\title{
2-D MHD CONFIGURATIONS FOR ACCRETION DISKS AROUND MAGNETIZED STARS
}

\author{
R. BENINI \\ ICRA - International Center for Relativistic Astrophysics \\ Dep. of Physics - "Sapienza" Università di Roma, Piazza A. Moro, 5 (00185), Roma, Italy \\ E-mail: riccardo.benini@icra.it \\ G. MONTANI \\ ENEA - C.R. Frascati (Unità Fus. Mag), Via E. Fermi, 45 (00044), Frascati (RM), Italy \\ ICRANet-C. C. Pescara, Piazzale della Repubblica, 10 (65100), Pescara, Italy \\ E-mail:montani@icra.it
}

We discuss basic features of steady accretion disk morphology around magnetized compact astrophysical objects. A comparison between the standard model of accretion based on visco-resistive MHD and the plasma instabilities, like ballooning modes, triggered by very low value of resistivity, is proposed.

Basic Model. The standard model of an axisymmetric thin disk is fixed by the fluid-dynamical equilibria established within the gravitational field of the compact central object (having mass $M_{S}$ ) when averaged out of the vertical direction. ${ }^{1}$ The angular frequency of the disk takes the Keplerian profile $\omega(r)=\omega_{K}=\sqrt{G M_{S} / r^{3}}$. Significant deviations from such a behavior are expected only in advective dominated regimes. The vertical equilibrium gives, for an isothermal disk of temperature $T$, the exponential decay of the mass density over the equatorial plane value $\epsilon_{0}(r)$, i. e. $D\left(z^{2}\right) \equiv \epsilon / \epsilon_{0}=\exp \left\{-z^{2} / H^{2}\right\}$, with $H=\sqrt{2 v_{s}^{2} / \omega_{K}^{2}}$ being the halfdepth of the disk and $v_{s}$ the sound velocity on the equatorial plane, that is to say $v_{s}^{2}=2 K_{B} T / m_{i}$ (here $K_{B}$ denotes the Boltzmann constant, while $m_{i}$ stands for the ions mass). The azimuthal equilibrium describes the angular momentum transport across the disk, by virtue of a turbulent viscosity coefficient $D_{\mu}^{0}$, i. e. $\dot{M}_{d}\left(L-L_{0}\right)=3 \pi D_{\mu}^{0} \omega_{K} r^{2}$, where $L$ is the angular momentum per unit mass, $L_{0}$ a fixed value and $\dot{M}_{d}=-2 \pi r \sigma v_{r}$ is the mass accretion rate, associated to the radial velocity $v_{r}<0$ and to the surface mass density $\sigma \equiv \int_{-H}^{H} \epsilon d z$. Finally the continuity equation implies that $\dot{M}_{d}=$ const $>0$.

The viscosity Coefficient. The viscosity coefficient $D_{\mu}^{0}$ that arises in the disk, as estimated by the microscopic plasma structure, results to be too small to account for the accretion rates observed in some astrophysical systems, like X-ray binaries. 
In fact, the observed accretion rates, evaluated by the increasing disk luminosity $\dot{L}_{d} \sim G \dot{M}_{d} M_{S} / R_{S}$, require a large value of $D_{\mu}^{0}$, that in ${ }^{2}$ were postulated to be due to a turbulent behavior of the disk plasma. Since, by definition $L=\omega r^{2}$, we can infer that $D_{\mu}^{0}=2 \sigma v_{t} H / 3, v_{t}$ being a turbulence velocity expressible as $v_{t}=\alpha v_{s}, \alpha$ being a free parameter. The point is that the axisymmetric disk is linearly stable with respect to small perturbations preserving its symmetry. For a discussion of the onset of turbulence by magnetohydrodynamics (MHD) instabilities based on the Velikov analysis of 1959 , see the review article. ${ }^{3}$ In what follows, we address a different point of view, based on the idea that the accretion phenomenon is related to the structures rising within the plasma disk.

MHD Disk Configuration. The magnetic field, characterizing the central object, takes the form $r \vec{B}=\partial_{z} \psi \vec{e}_{r}+I \vec{e}_{\phi}+\partial_{r} \psi \vec{e}_{z}$ with $\psi=\psi\left(r, z^{2}\right)$ and $I=I(\psi, z)$. The matter flux within the disk reads as

$$
\epsilon \vec{v}=-\frac{1}{r} \partial_{z} \Theta \vec{e}_{r}+\epsilon \omega\left(r, z^{2}\right) r \vec{e}_{\phi}+\frac{1}{r} \partial_{r} \Theta \vec{e}_{z}
$$

where $\Theta(r, z)$ has to be an odd function of $z$ to deal with a non-zero accretion rate.

A local model of the equilibrium, ${ }^{4-6}$ around a radius value $r=r_{0}$, allows to study the effects of the electromagnetic reaction of the disk plasma. We split the energy density and the pressure as $\epsilon=\bar{\epsilon}\left(r_{0}, z^{2}\right)+\hat{\epsilon}$ and $p=\bar{p}\left(r_{0}, z^{2}\right)+\hat{p}$, respectively. As well, the magnetic surface function splits in the form $\psi=\psi_{0}\left(r_{0}\right)+\psi_{1}\left(r_{0}, r-r_{0}, z^{2}\right)$, with $\psi_{1} \ll \psi_{0}$. The corotation theorem ${ }^{7}$ states that $\omega=\omega(\psi)$. Hence, we can take the decomposition $\omega=\omega_{K}+\omega_{0}^{\prime} \psi_{1}$, where $\omega_{0}^{\prime}=$ const. We now define the dimensionless functions $Y, \hat{D}$ and $\hat{P}$, as follows

$$
Y \equiv \frac{k_{0} \psi_{1}}{\partial_{r_{0}} \psi_{0}}, \quad \hat{D} \equiv \frac{\beta \hat{\epsilon}}{\epsilon_{0}}, \quad \hat{P} \equiv \beta \frac{\hat{p}}{p_{0}},
$$

where $p_{0} \equiv 2 K_{B} \hat{T} \epsilon_{0} / m_{i}$ and $\beta \equiv 8 \pi p_{0} / B_{0 z}^{2}=1 /\left(3 \epsilon_{z}^{2}\right) \equiv k_{0}^{2} H_{0}^{2} / 3$. Here $k_{0}$ is the fundamental wavenumber of the radial equilibrium, defined as $k_{0} \equiv 3 \omega_{K}^{2} / v_{A}^{2}$, with $v_{A}^{2} \equiv 4 \pi \epsilon_{0} / B_{z 0}^{2}$, recalling that $B_{z 0}=\partial_{r_{0}} \psi_{0} / r_{0}$. Thus, we deal with the dimensionless radial and vertical variables $x \equiv k_{0}\left(r-r_{0}\right)$ and $u \equiv z /\left(\sqrt{\epsilon_{z}} H_{0}\right)$, respectively. The vertical and radial equilibria can be restated as follows

$$
\begin{gathered}
\partial_{u^{2}} \hat{P}+\epsilon_{z} \hat{D}+2\left(\partial_{x^{2}}^{2} Y+\epsilon_{z} \partial_{u^{2}}^{2} Y\right) \partial_{u^{2}} Y=0 \\
\left(D+\frac{1}{\beta} \hat{D}\right) Y+\partial_{x^{2}}^{2} Y+\epsilon_{z} \partial_{u^{2}}^{2} Y+\frac{1}{2} \partial_{x} \hat{P}+\left(\partial_{x^{2}}^{2} Y+\epsilon_{z} \partial_{u^{2}}^{2} Y\right) \partial_{x} Y=0
\end{gathered}
$$

The electron force balance equation. In the presence of a non-zero resistivity coefficient $\eta$, the equation of the electron force balance, reads as: $\vec{E}+\frac{\vec{v}}{c} \wedge \vec{B}=\eta \vec{J}$, $\vec{E}$ denoting the electric field and $\vec{J}$ the current density. Since the axial symmetry requires $E_{\phi} \equiv 0$, in the local formulation around $r_{0}$, the azimuthal component stands as

$$
v_{r} B_{z}-v_{z} B_{r}=\frac{\eta c^{2}}{4 \pi r_{0}}\left(\partial_{r}^{2} \psi_{1}+\partial_{z}^{2} \psi_{1}\right)
$$


The discussion about the microscopic viscosity coefficient can be directly extrapolated for the resistivity coefficient too. Thus, for instance, in the linear regime $B_{r} \sim 0$, the radial matter infall associated to the dissipative term balancing the electron equilibrium, can not provide a sufficient accretion rate. Therefore, we are lead to argue that the azimuthal electron force balance stands on average as $\left\langle v_{r} B_{z}-v_{z} B_{r}\right\rangle \simeq 0$. The driving force of the mass accretion must be searched in the peculiar MHD instabilities arising in the plasma as triggered by very small resistivity contributions, like the so-called resistive ballooning modes. ${ }^{8}$ Eqs. 344 admit, at lowest order in $\epsilon_{z}$, a solution which gives the relevant magnetic surfaces in the form

$$
Y_{t} \simeq x-Y_{0}^{0} \sin x F_{0}\left(u^{2}\right), \quad Y_{0}^{0}=\mathrm{const}
$$

We argue that in the region around $r_{0}+\pi / k_{0}$ the magnetic field lines are crowded between two adjacent separatrices and the flow across them can be thought as being maintained intermittently by recurrent resistive ballooning modes. Such modes, driven by the combined effect of the local radial density gradient and of gravity, let the plasma slip through the magnetic field lines (as sketched in Fig. 1).

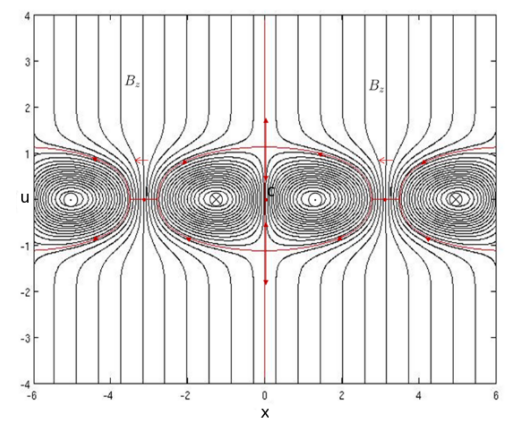

Fig. 1. This picture sketches the plasma along the separatrix.

This work was developed within the framework of the CGW Collaboration (www.cgwcollaboration.it) and the authors would like to thank Bruno Coppi for his valuable advice on the topic and for providing us with the Fig. 1

\section{References}

1. G. S. Bisnovatyi-Kogan and R. V. E. Lovelace, New Astronomy Review 45, 663 (2001).

2. N. I. Shakura, Soviet Astronomy 16, 756 (1973).

3. S. A. Balbus and J. F. Hawley, Reviews of Modern Physics 70, 1 (1998).

4. B. Coppi, Physics of Plasmas 12, 057302 (2005).

5. B. Coppi and F. Rousseau, The Astrophysical Journal 641, 458 (2006).

6. G. Montani and R. Benini, to appear on Modern Physics Letters A (2009).

7. V. C. A. Ferraro, Monthly Notices of the Royal Astronomical Society 97, 458 (1937).

8. B. Coppi, Physical Review Letters 39, 939 (1977). 